Article

\title{
Long-Term Effects of a Soft Robotic Suit on Gait Characteristics in Healthy Elderly Persons
}

\author{
Shanhai Jin ${ }^{1} \mathbb{C}$, Xiaogang Xiong ${ }^{2, *} \mathbb{C}$, Dejin Zhao ${ }^{1}$, Changfu Jin ${ }^{1}$ and Motoji Yamamoto ${ }^{3, *}$ \\ 1 School of Engineering, Yanbian University, Yanji 133002, China; jinshanhai@ybu.edu.cn (S.J.); \\ djzhao@ybu.edu.cn (D.Z.); jincf9@163.com (C.J.) \\ 2 School of Mechanical Engineering and Automation, Harbin Institute of Technology (Shenzhen), \\ Shenzhen 518055, China \\ 3 Faculty of Engineering, Kyushu University, Fukuoka 8190395, Japan \\ * Correspondence: xiongxg@hit.edu.cn (X.X.); yama@mech.kyushu-u.ac.jp (M.Y.)
}

Received: 10 March 2019; Accepted: 8 May 2019; Published: 13 May 2019

\begin{abstract}
As a walking assistive device for elderly persons, one of the major aims should be to improve and rehabilitate gait characteristics after long-term repeated use of the device. However, most of the existing research on walking assistive devices only emphasize their immediate effects, and there is limited research indicating the long-term effects. To address this gap, this paper experimentally validates the effects of our soft wearable robotic suit on gait characteristics of elderly persons after repeated use of the device for six weeks. Experimental results on four elderly subjects (age $=74.8 \pm 5.0$ year) show that, after six weeks of gait rehabilitation training by the robotic suit, the gait characteristics of the subjects were improved, leading to an increased walk ratio with an average of $9.8 \%$ compared with the initial state. The results of this research will benefit the potential use of the robotic suit in gait training and rehabilitation for elderly persons and also will be useful to the establishment of practical guidelines that maximize the training and rehabilitation effectiveness of the robotic suit.
\end{abstract}

Keywords: rehabilitation robotics; soft walking assistive robot; long-term effect; gait characteristics; elderly person

\section{Introduction}

The worldwide elderly population has increased to $12.3 \%$, and the number will increase dramatically to an estimated $22 \%$ by the year 2050 [1,2]. One of the major age-related changes is the decline of walking performance that is featured by slow walking speed, rapid cadence, reduced step length, and decreased joint range of motion, as a consequence of lower limb skeletal muscle degradation [3-7]. Such declining walking performance may result in reduced daily physical activities, which in turn probably cause further muscle degeneration of the lower limbs. Finally, elderly persons may fall into a vicious cycle of skeletal muscle decline and daily physical activity reduction. Thus, for elderly persons, rehabilitating or at least maintaining walking performance is appealing.

In order to prevent this kind of vicious cycle, researchers have developed lower limb exoskeletons. For example, Cyberdyne Inc. [8] researched a walking-function-regaining exoskeleton. Honda Motor Co., Ltd. [9] studied a walking assistive device for improving walking ability in daily activities, and IHMC [10] presented a robotic orthosis for hip and knee assistance, as other examples. Besides these, many similar exoskeletons have also been reported in the literature [11-16]. One of the major advantages of these devices is that they are capable of generating large enough forces for assisting the human joints. Besides that, the full or a greater portion of the body weight of wearers can be shared by their rigid frames. Even so, before their practical applications, some critical issues regarding 
their design and implementation should be solved. 1. Joint misalignment between exoskeleton and wearer may result in resistive force [17] and, consequently, may result in increase of energy expenditure. 2. Rigid frames may constrain the motion range of the lower limb of the wearer [18], while conducting daily physical activities requires a sufficient motion range. 3. A massive power supply system is required for generating a large assistive force. This places additional payload on the wearer and also may lead to increased energy expenditure. 4. The procedures of mounting and removing an exoskeleton are complicated, even impossible in some cases without the help of a fully-trained assistant. Such issues perhaps are problematic when using exoskeletons in daily physical activities.

To avoid the limitations of exoskeletons, soft robotic devices have been researched for lower limb assistance, among which typical ones include Park et al.'s soft orthosis for ankle assistance $[19,20]$, Asbeck et al.'s soft exosuit for ankle assistance [21], Ding et al.'s multi-joint soft exosuit for gait assistance [22,23], and our soft wearable robotic suit for hip assistance [24,25]. In such devices, exact joint alignment between wearer and device is not necessary. Additionally, because of the adoption of soft materials, wearers can walk with almost no kinematic constraints, and they can conduct interactions with devices in a biomechanically-compatible and safe way [26]. Moreover, a smaller and lighter power supply system can be applied because they usually provide relatively less assistive power compared with exoskeletons.

Specifically, in previous works [24,25], the author and his colleagues have presented a soft robotic suit, as shown in Figure 1, for walking assistance for elderly persons in daily physical activities. The device provides a small, yet effective assistive force for hip flexion. In addition, its weight is light, and it provides an almost full lower limb motion range for wearers. It has been reported [24] that, as an immediate effect on nine $74.2 \pm 3.7$ year-old elderly persons, the device significantly reduced energy expenditure in level walking with an average of $5.9 \%$ and significantly increased the walk ratio, which is a normalized measure of step length (i.e., stride length divided by cadence) [27], with an average of $8.9 \%$ in the condition of the device worn and powered on (PON) compared with the condition of worn, but powered off (POFF). It has also been reported [25] that energy expenditure was significantly reduced by $6.5 \%$ for inclined walking in the PON condition compared with the POFF condition.

One of the major aims of walking assistive devices should be to provide gait training and rehabilitation for elderly persons by repeated use of the device. However, most of the existing research only focuses on immediate effects of the devices, e.g., the differences between the POFF condition and the PON condition. There is limited research indicating the long-term effects, e.g., the differences of the POFF condition between the initial state and the state of after long-term repeated use, and only a few reports are available in the literature [28]. To address this gap, this paper experimentally validates the effects of our robotic suit on gait characteristics after six weeks' repeated use of the device for demonstrating its potential use in gait training and rehabilitation for elderly persons. It is hoped that the results of this research will be useful to the establishment of practical guidelines that maximize the training and rehabilitation effectiveness of the device.

The rest of the paper is provided as follows. An overview of the author and his colleagues' soft robotic suit is given in Section 2. The six weeks' effects of the device on gait characteristics are experimentally validated in Section 3. Section 5 discusses the experimental results, and Section 6 concludes the paper and suggests future work. 


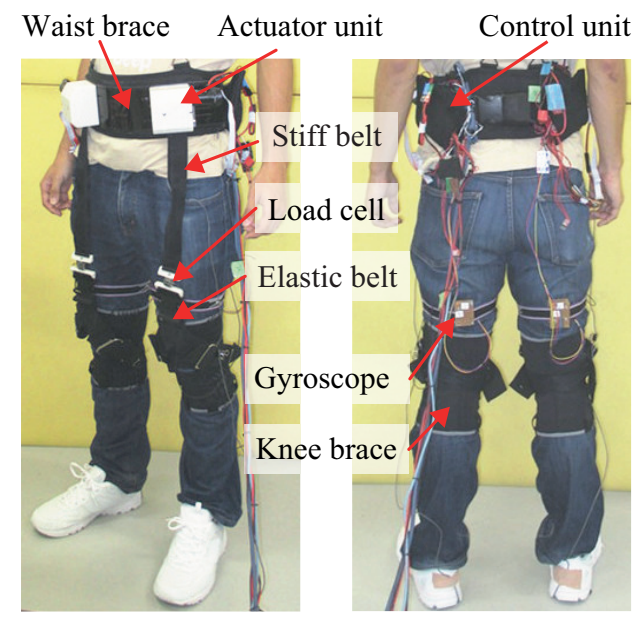

Figure 1. Entire design of a soft wearable robotic suit for hip flexion.

\section{Overview of a Soft Wearable Robotic Suit}

We have presented a soft wearable robotic suit for energy-efficient walking for elderly persons in daily physical activities. Figure 1 illustrates the entire design of the device, which consists of a waist support brace, knee braces, actuators, controllers, stiff and elastic belts, load cells, and gyroscopes. For each leg, one actuator and one controller were affixed to the front and the back sides of the waist support brace, respectively. The upper end of the stiff belt was pinned to the actuator, and the bottom two ends of the inverted Y-shaped elastic belt were attached to the lateral two sides of the knee brace, respectively. In addition, the bottom end of the stiff belt and the upper end of the elastic belt were connected through the load cell. Moreover, the gyroscope was attached to the thigh. The total mass of the device was $2.7 \mathrm{~kg}$, excluding the power supply system (the power of the device was supplied by an external power supply by a power cable.).

The robotic suit assists hip flexion through a small, yet effective tension force, as shown in Figure 2. Specifically, a tension force is generated on the stiff and elastic belts by the winding up of the belts during the swing phase, and it is transmitted to the wearer's hip joint. In the case of stand phase, the device provides a small enough tension force that permits "creeping" of the belts along the thigh without influencing the hip extension.

Figure 3 shows the control architecture. The hip angle was calculated through the numerical integration of the hip angular velocity, which was obtained by the gyroscope. Estimations of average gait cycle and three events of heel contact, minimum hip angle, and maximum hip angle were conducted. Then, these estimated values were applied for the desired assistive force generation. In order to track the desired force smoothly and safely, a proxy-based sliding mode controller [29] was conducted with a control cycle of $T=0.001 \mathrm{~s}$. Figure 4 illustrates the typical data of hip angle and assistive force.

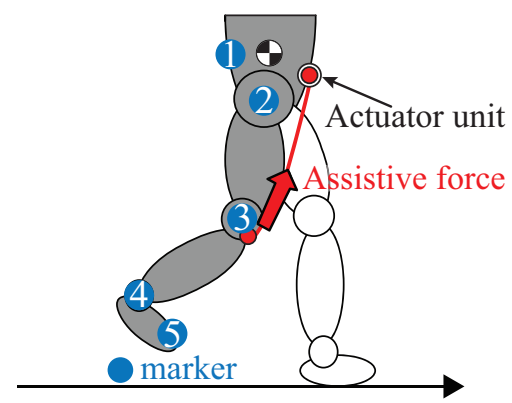

Figure 2. Assistance strategy for hip flexion. Blue-filled circles express reflective markers attached to the subjects in the experiment of Section 3 (1. approximation of the center of mass, 2. hip, 3. knee, 4. ankle, and 5. toe). 


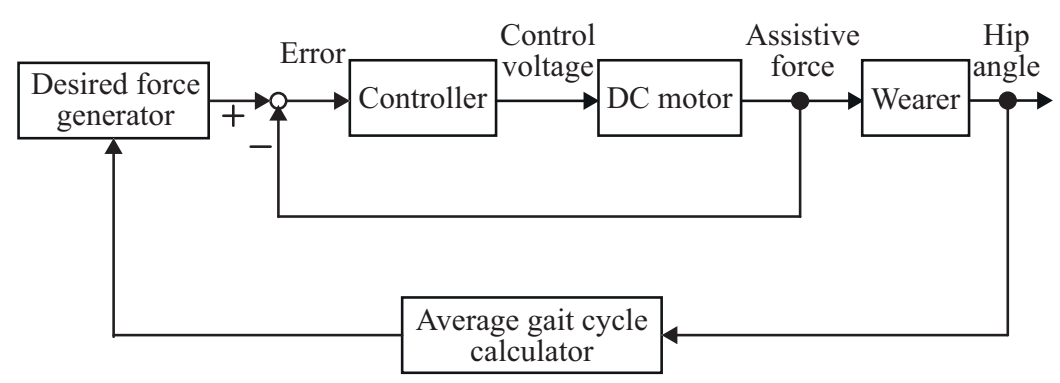

Figure 3. Control architecture of the robotic suit.
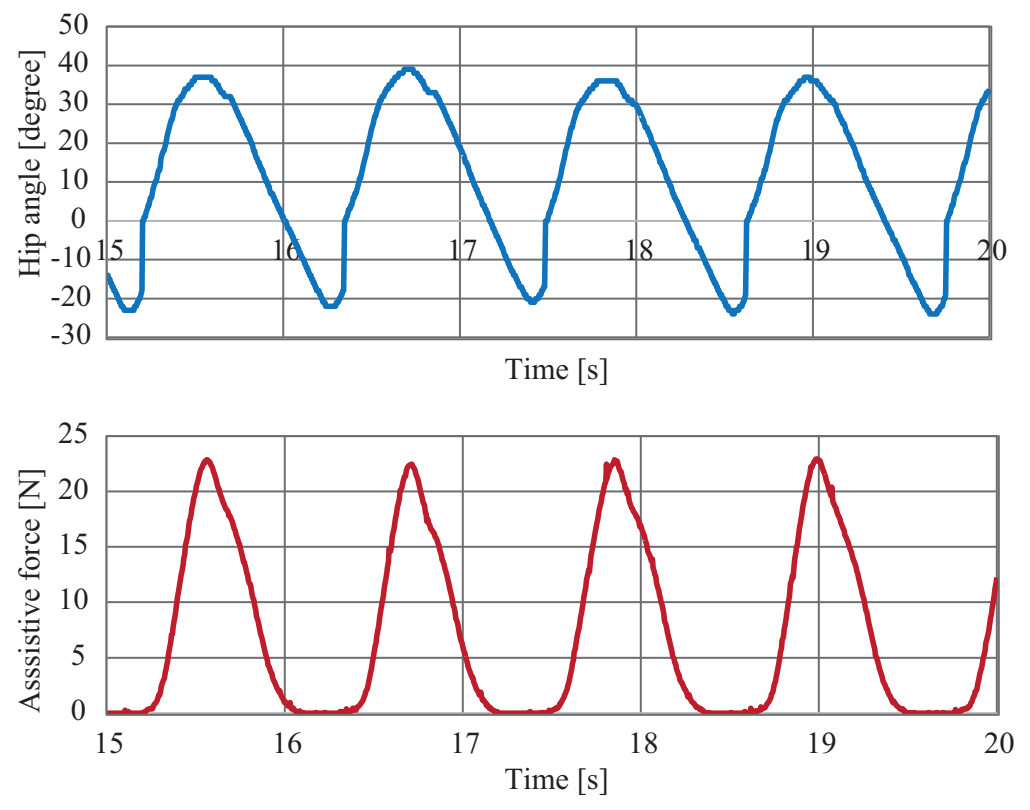

Figure 4. Measured hip angle and generated assistive force.

\section{Experiment}

A 6-week experiment was conducted for validating the long-term effects of the robotic suit on the gait characteristics of elderly persons. Here, it should be mentioned that the term "long-term effects" is a relative term. Specifically, it refers to the differences of the POFF condition between the initial state and the state of after 6 weeks' repeated use of the robotic suit and is used to distinguish it from "immediate effects", which refers to the comparison between the POFF condition and the PON condition. The Experiment Ethics Committee of the Faculty of Engineering of Kyushu University approved the experiment.

\subsection{Subjects}

Four healthy elderly subjects (two males, two females, age $=74.8 \pm 5.0$ years, weight $=60.0 \pm 9.2 \mathrm{~kg}$, height $=160.5 \pm 7.5 \mathrm{~cm}$ ) participated in the experiment.

\subsection{Protocol}

Each subject performed a preliminary exercise before conducting the main experiment. First, subjects were allowed to walk on a treadmill (SportsArt, FM-TR22F) for familiarizing with treadmill walking. Then, the procedure reported in [30] was applied for determining the preferred walking speed of each subject $(3.8 \pm 0.5 \mathrm{~km} / \mathrm{h})$. After that, at the preferred walking speed, each subject was instructed to walk in the PON condition with the maximum assistive force $23.3 \pm 4.9 \mathrm{~N}$ for getting use to the device. Here, for each subject, the maximum assistive force was set as the value that the subject felt most comfortable with in the PON condition by considering individual differences [31]. Moreover, 
for all subjects, the assistance started at the event of minimum hip angle, reached its maximum value at the event of maximum hip angle, and ended at the event of heel contact.

The evaluation was conducted continuously over a period of 6 weeks. Each subject participated in the experiment at the same time (e.g., 9:00) on the same day (e.g., Monday) of each week. On each experimental day, each subject performed 4 trials of 6-min treadmill walking at the preferred walking speed, as shown in Figure 5. Among them, two trials were in the POFF condition, and the other two were in the PON condition. A 10-min rest was conducted after Trials 1 and 3, and a 20-min rest was provided after Trial 2. For excluding the influences of measurement errors and biomechanical variations, the order of the experimental conditions was reversed on each week. Specifically, for each subject, the trial order was randomly determined for Week 1 (i.e., either Trial Order A or Trial Order B, as depicted in Figure 5). Then, for the remaining 5 weeks, the reverse order of each previous week was applied.

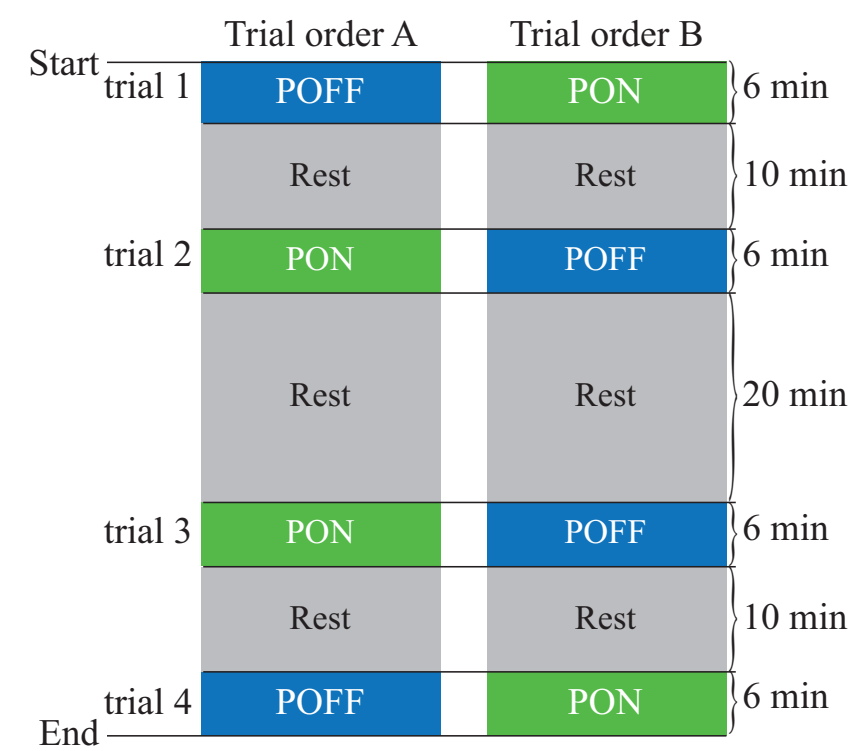

Figure 5. Protocol of each experimental day. PON, powered on; POFF, powered off.

For each trial, as illustrated in Figure 2, reflective markers were affixed to an approximation of the center of mass (COM), hip, knee, ankle, and toe. A 6-camera motion capture system (S250e, NaturalPoint, Inc., Oregon, USA) was used for tracking the reflective markers during the last 1-min period of walking with a frequency of $75 \mathrm{~Hz}$.

\subsection{Data Analysis}

Missing marker frames (maximum 10 frames) of the measured data were filled by applying a third-degree polynomial interpolation provided by the Motive software (NaturalPoint, Inc.). Horizontal position data of the markers were measured in absolute coordinates, and then, they were converted to the relative coordinate data by setting the horizontal position of COM in the absolute coordinate as the horizontal origin of the relative coordinate system.

For all trials, joint angles were calculated by using the kinematic data. In addition, stride duration was obtained by detecting the period between two successive maximum horizontal positions of the toe. Then, cadence was computed by using the stride duration, and step length was calculated from cadence and walking speed. After that, the walk ratio was obtained by dividing the step length by cadence. The averages of all trials of each subject in each condition of each day were used for the analysis.

Paired $t$-tests were conducted for identifying the significant changes of maximum hip angles (flexion and extension), maximum knee angles (flexion and extension), maximum ankle angles 
(dorsiflexion and plantar flexion), and the walk ratio (i.e., stride length divided by cadence) in the POFF condition between Week 1 and Week 6 . In addition, a paired $t$-test was also performed to determine the significant difference in the walk ratio between the POFF condition and the PON condition by using the average value of four subjects in each condition of each week. Standard deviations were calculated for all measures.

\section{Results}

Figure 6 compares average maximum hip, knee, and ankle angles of each week between the POFF condition and the PON condition. It is shown that the effects of assistance were mainly detected in hip flexion, knee flexion, and ankle dorsiflexion, with average increases of $10.5 \%, 1.9 \%$, and $33.7 \%$, respectively. In addition, the tendency of the values increased as the week progressed.

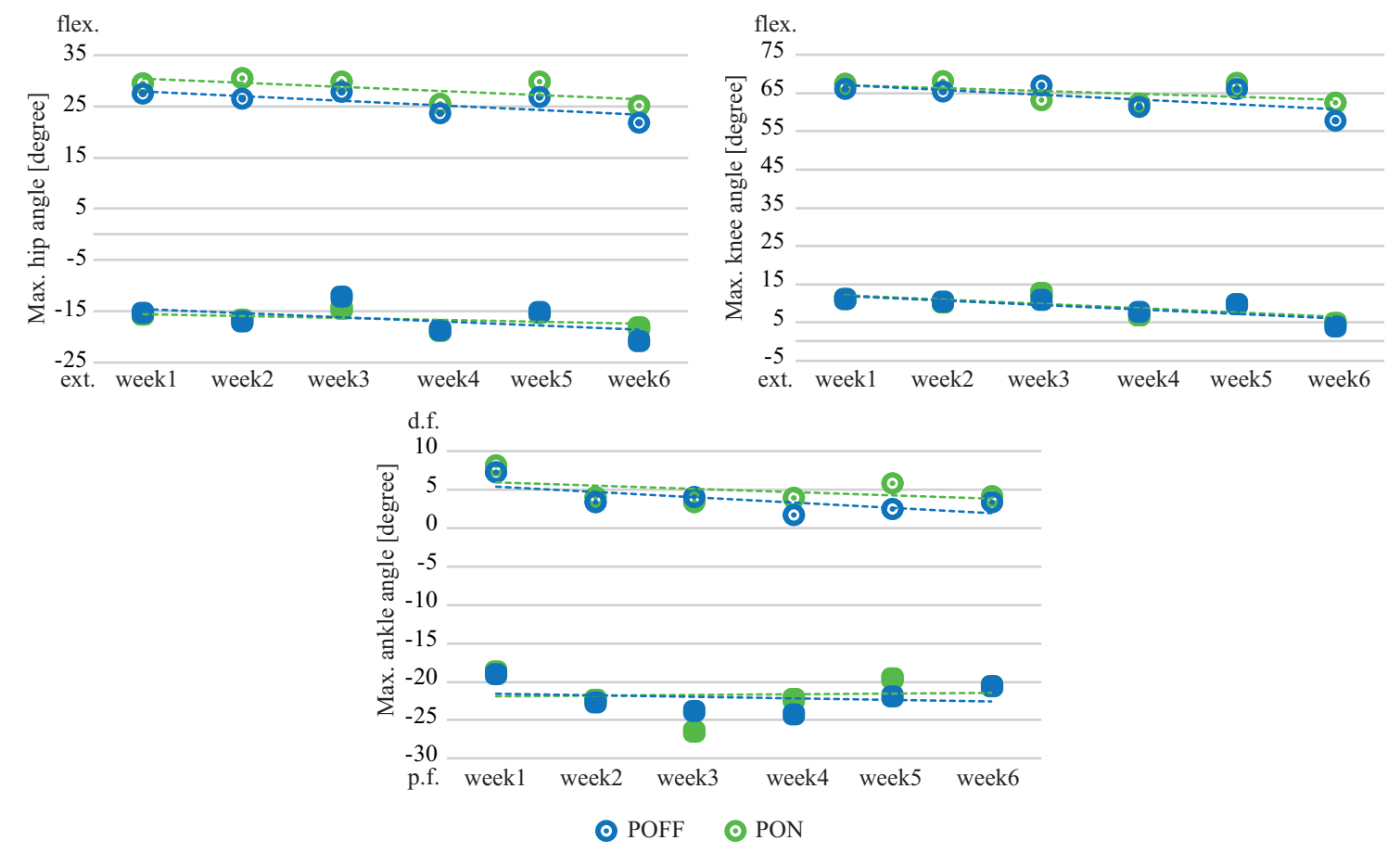

Figure 6. Comparison of average maximum joint angles of each week between the POFF condition and the PON condition. Here, flex.: flexion, ext.:extension, d.f.: dorsiflexion, and p.f.: plantar flexion.

Figures 7 and 8 compare the joint angles of hip, knee, and ankle achieved in the POFF condition between Week 1 and Week 6. It was observed that, after six weeks' use of the robotic suit, the gait characteristics were changed. Specifically, maximum hip flexion angle was reduced from $27.6 \pm 3.1$ degrees to $22.5 \pm 3.2$ degrees, while maximum hip extension angle was increased from $15.4 \pm 9.1$ degrees to $20.8 \pm 5.2$ degrees. For the knee joint, maximum extension angle reached $3.6 \pm 3.9$ degrees from $10.1 \pm 9.0$ degrees, while maximum flexion angle reduced to $58.3 \pm 6.2$ degrees from $66.5 \pm 5.0$ degrees. Besides that, in the case of ankle joint, the maximum dorsiflexion angle was reduced to $3.4 \pm 5.8$ degrees from $7.4 \pm 5.0$ degrees, while maximum plantar flexion angle was increased to $21.4 \pm 5.6$ degrees from $19.4 \pm 6.1$ degrees. Statistically-significant differences were found in maximum hip flexion, maximum knee flexion, and maximum ankle dorsiflexion, with an average reduction of $20.7 \%, 12.5 \%$, and $54.3 \%$, respectively, in Week 6 . 

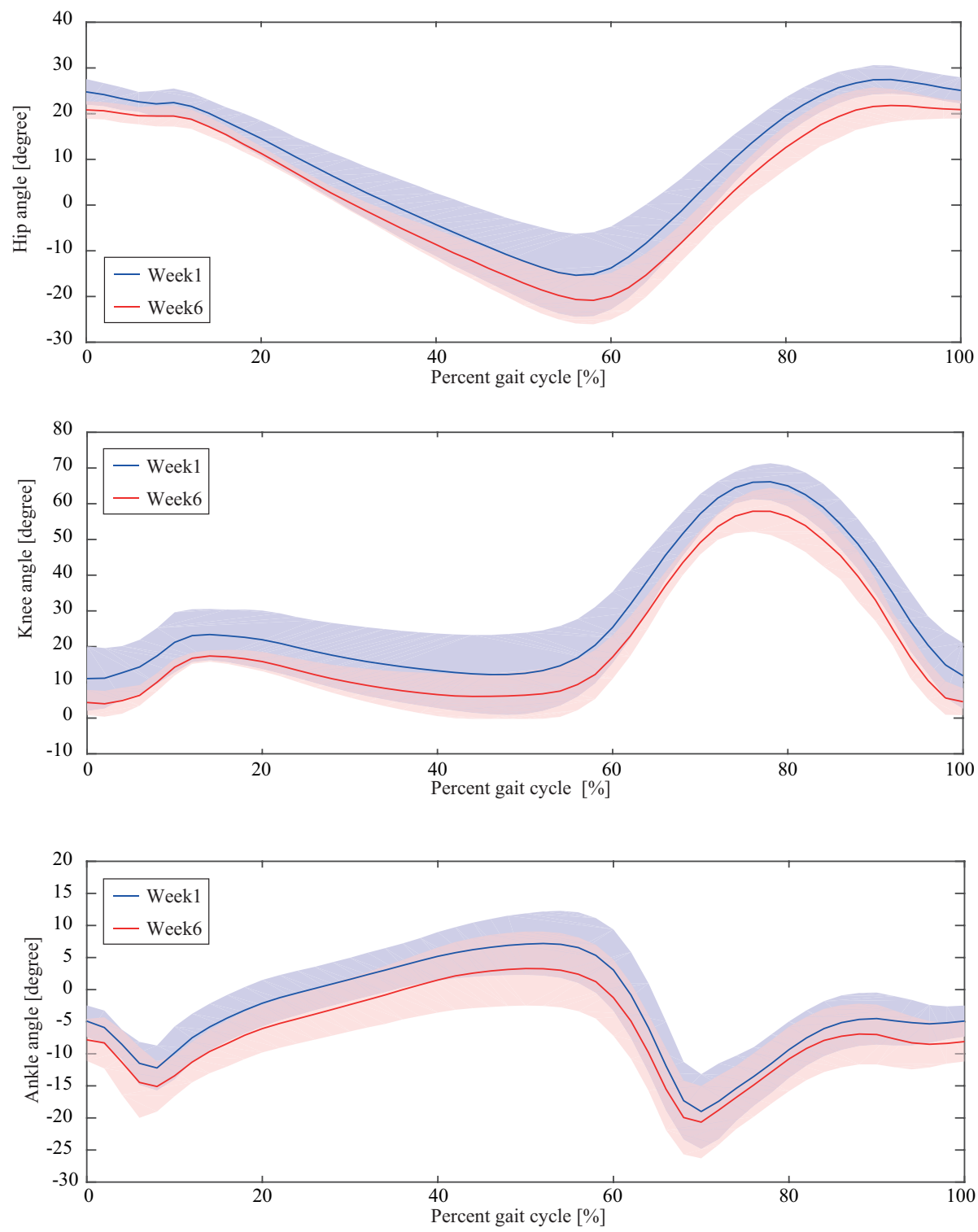

Figure 7. Comparison of joint angles achieved in the POFF condition between Week 1 and Week 6. On each graph, the solid curve represents the average value, and the shadowed region denotes one standard deviation.
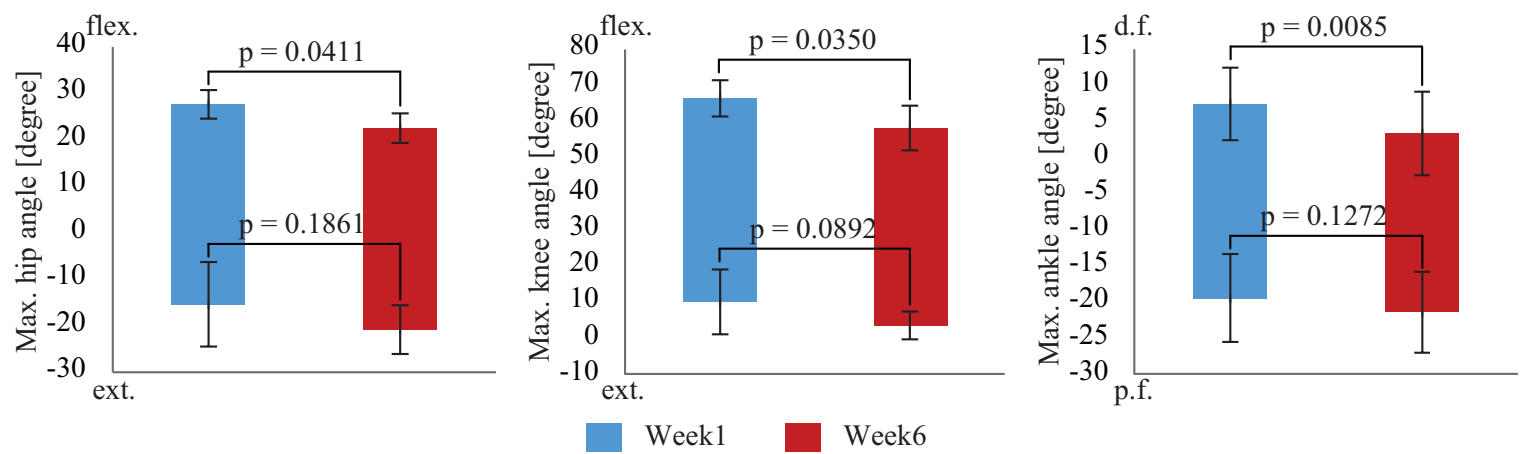

Figure 8. Comparison of maximum joint angles achieved in the POFF condition between Week 1 and Week 6. Here, flex.: flexion, ext.:extension, d.f.: dorsiflexion, and p.f.: plantar flexion. On each bar chart, the error bar denotes one standard deviation. 
As a result of the above-mentioned changes, the walk ratio varied concurrently, as illustrated in Figure 9. It is shown that, for each week, the walk ratio of the PON condition was higher than that of the POFF condition. In addition, the tendencies of both conditions increased as the week progressed (POFF: $y=0.00009148 x+0.00503251, R^{2}=0.623$; PON: $y=0.00004885 x+0.00555119, R^{2}=0.397$ ), but the difference between the two conditions decreased.

Figure 10 shows statistical comparisons of the six weeks' average walk ratio between the POFF condition and the PON condition. It is shown that walk ratio of the PON condition was $0.0057 \mathrm{~m} / \mathrm{steps} /$ $\mathrm{min}$, while that of the POFF condition was $0.0054 \mathrm{~m} / \mathrm{steps} / \mathrm{min}$. A statistically-significant difference was detected.

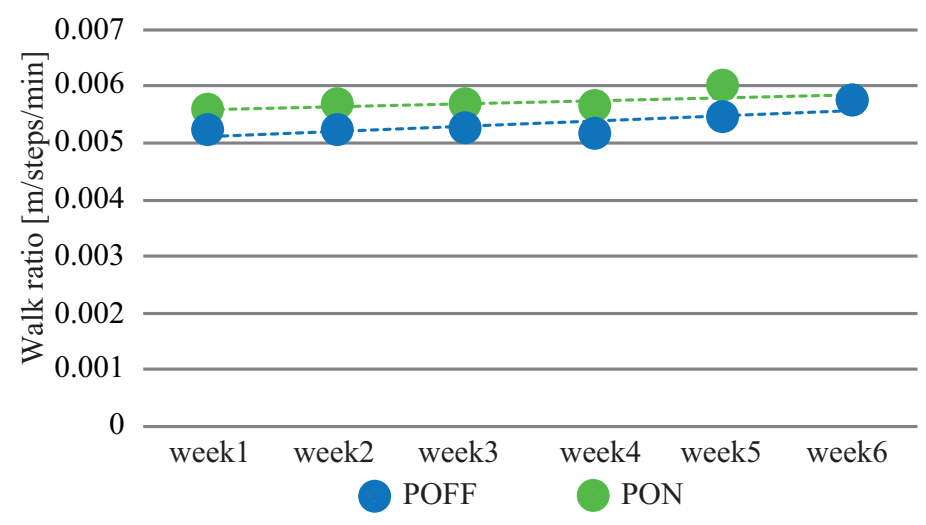

Figure 9. Comparison of the average walk ratio of each week between the POFF condition and the PON condition.

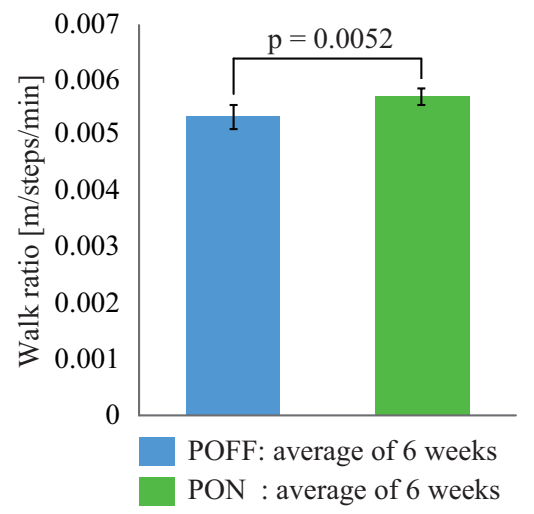

Figure 10. Comparison of the average walk ratio between the POFF condition and the PON condition.

On each bar chart, the error bar denotes one standard deviation.

Figure 11 compares the individual walk ratio of the POFF condition between Week 1 and Week 6 . It is shown that, compared with initial state, the walk ratio of three subjects increased $6.8 \%, 22.2 \%$, and $13.7 \%$, respectively, after six weeks' use of the robotic suit, while that of one subject slightly decreased $2.9 \%$. Figure 12 shows statistical comparisons of the walk ratio achieved in the POFF condition between Week 1 and Week 6 . It is shown that, although no significant difference was detected, after six weeks, the walk ratio increased from $0.0052 \pm 0.0013 \mathrm{~m} / \mathrm{steps} / \mathrm{min}$ to $0.0057 \pm 0.0009 \mathrm{~m} / \mathrm{steps} / \mathrm{min}$ in the POFF condition, with an average increase of $9.8 \%$.

Figure 13 demonstrates typical motions around the timings of the toe off and the heel contact. One can observe that, compared with Week 1 , the lower limb of Week 6 acted more like an inverted pendulum motion. 


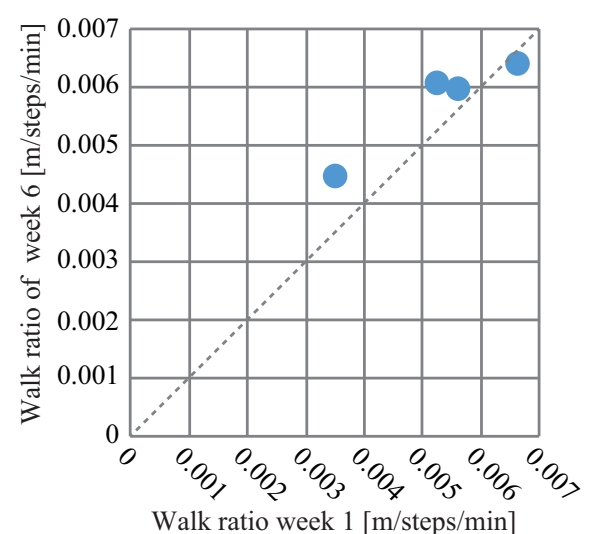

Figure 11. Comparison of the walk ratio of each subject achieved in the POFF condition between Week 1 and Week 6.

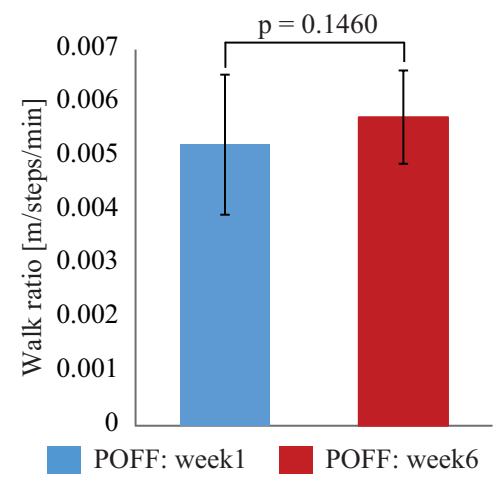

Figure 12. Comparison of the walk ratio achieved in the POFF condition between Week 1 and Week 6. On each bar chart, the error bar denotes one standard deviation.

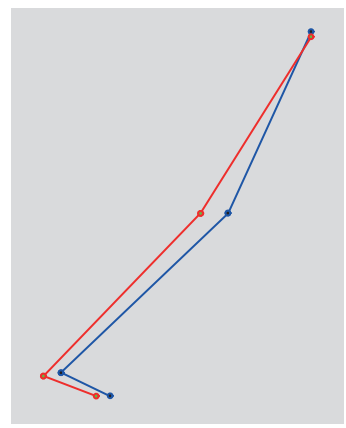

Gait cycle $56 \%$

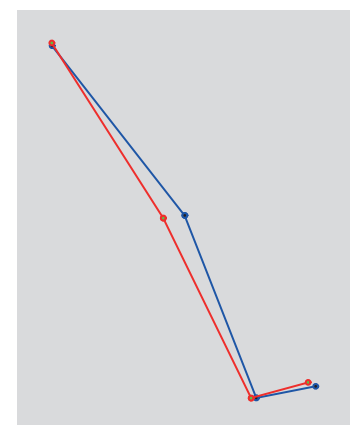

Gait cycle $100 \%$

- Week 1

Figure 13. Comparison of the typical motions of the POFF condition around the timings of the toe off and the heel contact between Week 1 and Week 6 .

\section{Discussion}

Motion ranges of hip, knee, and ankle joints increased in the PON condition compared with those in the POFF condition. Correspondingly, the walk ratio of the PON condition increased to $0.0057 \mathrm{~m} / \mathrm{steps} / \mathrm{min}$ from $0.0054 \mathrm{~m} / \mathrm{steps} / \mathrm{min}$ of the POFF condition, with an average increase of $6.9 \%$. Toward this, we considered that the provided hip flexion assistance contributed to the hip flexion and consequently brought the swing leg to be positioned further forward compared with the case of the POFF condition. This result is consistent with our previous results [24] showing that the walk ratio in the PON condition was significantly increased to $0.0057 \mathrm{~m} / \mathrm{steps} / \mathrm{min}$ with an average increase of $8.9 \%$ compared with that in the POFF condition. It is recognized that healthy young persons walk at a walk 
ratio of approximately $0.0065 \mathrm{~m} / \mathrm{steps} / \mathrm{min}$ [32]. Thus, it can be confirmed that, as immediate effects (i.e., comparison between the POFF condition and the PON condition), the robotic suit improved gait characteristics of the elderly persons to a level closer to a healthy gait pattern.

On the other hand, after six weeks' use of the robotic suit, the walk ratio in the POFF condition reached $0.0057 \pm 0.0013 \mathrm{~m} / \mathrm{steps} / \mathrm{min}$ from its initial state $0.0052 \pm 0.0009 \mathrm{~m} / \mathrm{steps} / \mathrm{min}$, with an average increase of $9.8 \%$. Moreover, it was interestingly observed that elderly persons with a lower walk ratio may improve more by repeated use of the robotic suit. A similar study has also been conducted by Shimada et al. [28] to evaluate the long-term effects of a hip assistive device for three months. However, they reported that there was no increase of the walk ratio after three months' walking training with their device, while they found a significant increase of the walk ratio in an experiment for validating the immediate effects [33]. Thus, it can be argued that, as long-term effects (i.e., comparison in the POFF condition between Week 1 and Week 6), our device had the potential to make a contribution to gait rehabilitation of elderly persons.

However, it is also found that, different from the immediate effect of the improved walk ratio by mainly increasing hip flexion, knee flexion, and ankle dorsiflexion, the walk ratio increased with reduced hip flexion, knee flexion, and ankle dorsiflexion, but increased hip extension, knee extension, and ankle plantar flexion in the case of long-term effects. Toward such a difference, we considered that the provided hip flexion assistance may indirectly influence the dynamics of the lower limbs. The most potential contributor could be the improved pelvic rotation. It is known $[34,35]$ that the pelvic rotation plays an important role in determining step length or, equivalently, the walk ratio. That is, the greater pelvic rotation allowed for a larger step length. It was also reported [36-38] that elderly persons walk in degraded pelvic rotation and hip extension, resulting in a declined walk ratio and walking speed. By considering these, in the case of using the device, we assumed that hip flexion assistance promoted the forward pelvis rotation of the elderly subjects. In turn, this promotion concurrently enhanced the standing leg muscles that were used for stabilizing the oppositely-rotating trunk, as pointed out in [39]. In such a way, after six weeks' repeated use of the device, it seems that muscles used for hip extension were reactivated and then softened, consequently leading to improved maximum hip extension. On the other hand, this improvement contributed to the improved gait characteristics with the increased walk ratio.

In addition, it was studied that energy expenditure of elderly persons during walking is higher than that of young persons [40]. Several researches have suggested that the possible reasons for such increase are decreased motion range of lower limb joints and shortened step length (i.e., walk ratio) [41-44]. It is also known [43] that a full knee extension (i.e., maximum knee extension angle with zero degrees) allows the lower limbs to behave like an inverted pendulum motion, with which a healthy person at preferred speed (i.e., the walk ratio was approximately $0.0065 \mathrm{~m} / \mathrm{steps} / \mathrm{min}$ ) optimizes the potential energy and the kinetic energy exchange of COM, consequently leading to minimized energy expenditure. In our previous study [24], it has been observed that energy expenditure was significantly reduced $5.9 \%$ in the PON condition with a significant $8.9 \%$ increase of walk ratio. Thus, it can be inferred that, as immediate effects, the elderly subjects participating in this study probably walked more easily and comfortably with less energy in the PON condition owing to the improved gait characteristics, although the energy expenditure was not directly measured here. Similarly, as long-term effects, increased knee extension and increased walk ratio achieved after six weeks' repeated use of the device also probably improved energy efficiency of walking for the elderly subjects in the POFF condition.

As a whole, it can be concluded that the robotic suit is effective not only at improving gait characteristics as immediate effects, but also potentially useful for rehabilitating gait characteristics as long-term effects. 


\section{Conclusions and Future Work}

This paper has experimentally validated the long-term effects of a soft robotic suit for walking assistance for elderly persons in daily activities. Experimental results illustrated that, after six weeks' use of the device, the gait characteristics of the subjects were improved to a level closer to a healthy gait pattern in the POFF condition, leading to an increased walk ratio with an average increase of $9.8 \%$ compared with the initial state. As was discussed, the promoted forward pelvis rotation and the correspondingly improved extensions of hip and knee joints were probably the major contributors for the rehabilitated gait characteristics. Such long-term effects of our robotic suit support its potential use in gait training and rehabilitation for elderly persons.

It also should be mentioned here that, although average improvements were found in some gait parameters, e.g., walk ratio, no significant differences were detected in these parameters probably due to the limit of the sample size of subjects and day-to-day variations.

As future research, the performance evaluation of the robotic suit for a longer period, e.g., a period longer than six months, is desirable for further supporting the effectiveness of the device in the long term. Future research also should include the development of effective training guidelines for maximizing rehabilitation effectiveness and the design of an adaptive control scheme for different walking patterns with the aim of achieving maximized effectiveness.

Author Contributions: Data curation, C.J.; Funding acquisition, S.J., X.X. and M.Y.; Project administration, M.Y.; Validation, D.Z.; Writing-original draft, S.J.; Writing-review and editing, X.X.

Funding: This research was funded by the Research Foundation of Jilin Province Development and Reform Commission (Grant No. 2019C048-2), the Shenzhen Peacock Technical Innovation Funding (Grant No. KQJSCX20170726103546683), the Science \& Technology Development Foundation of Jilin Province (Grant No. 20180520019JH), the Science \& Technology Research Foundation of the Education Department of Jilin Province (Grant No. JJKH20180883KJ), and the Grant-in-Aid for the Adaptable \& Seamless Technology Transfer program through Target-driven R\&D of the Japan Science and Technology Agency (Grant No. AS2415010K).

Conflicts of Interest: The authors declare no conflict of interest.

\section{References}

1. United Nations Population Fund. Aging. 2015. Available online: Http:/ / www.unfpa.org/ageing (accessed on 13 May 2019).

2. Herrmann, M.; Guzman, J.M.; Juran, S.; Schensul, D. Population Dynamics in the Least Developed Countries: Challenges and Opportunities for Development and Poverty Reduction. 2011. Available online: http:/ / www.unfpa.org/publications/population-dynamics-ldcs (accessed on 13 May 2019).

3. Prince, F.; Corriveau, H.; Hebert, R.; Winter, D. Gait in the elderly. Gait Posture 1997, 5, 128-135. [CrossRef]

4. DeVita, P.; Hortobagyi, T. Age causes a redistribution of joint torques and powers during gait. J. Appl. Physiol. 2000, 88, 1804-1811. [CrossRef] [PubMed]

5. Watelain, E.; Barbier, F.; Allard, P.; Thevenon, A.; Angue, J. Gait pattern classification of healthy elderly men based on biomechanical data. Arch. Phys. Med. Rehabil. 2000, 81, 579-586. [CrossRef]

6. Menz, H.; Lord, S.; Fitzpatrick, C. Age-related differences in walking stability. Age Ageing 2003, 32, 137-142. [CrossRef] [PubMed]

7. Kirkwood, R.; Moreira, B.; Vallone, M.; Mingoti, S.; Dias, R.; Sampaio, R. Step length appears to be a strong discriminant gait parameter for elderly females highly concerned about falls: A cross-sectional observational study. Physiotherapy 2011, 97, 126-131. [CrossRef] [PubMed]

8. Yasuhara, K.; Shimada, K.; Koyama, T.; Ido, T.; Kikuchi, K.; Endo, Y. Walking assist device with stride management assist. Honda RED Techn. Rev. 2009, 21, 54-62.

9. Kawamoto, H.; Hayashi, T.; Sakurai, T.; Eguchi, K.; Sankai, Y. Development of single leg version of HAL for hemiplegia. In Proceedings of the 31st Annual International Conference of the IEEE Engineering in Medicine and Biology Society, Minneapolis, MI, USA, 2-6 September 2009; pp. 5038-5043.

10. Raj, A.K.; Neuhaus, P.D.; Moucheboeuf, A.M.; Noorden, J.H.; Lecoutre, D.V. Mina: A sensorimotor robotic orthosis for mobility assistance. J. Robot. 2011, 2011, 284352. [CrossRef] 
11. Yeh, T.; Wu, M.; Lu, T.; Wu, F.; Huang, C. Control of McKibben pneumatic muscles for a power-assist, lower-limb orthosis. Mechatronics 2010, 20, 686-697. [CrossRef]

12. Ikehara, T.; Tanaka, E.; Nagamura, K.; Tamiya, T.; Ushida, T.; Hashimoto, K.; Kojima, S.; Ikejo, K.; Yuge, L. Development of closed-fitting-type walking assistance device for legs with self-contained control system. J. Robot. Mech. 2010, 22, 380-390. [CrossRef]

13. Wu, Q.; Wang, X.; Du, F.; Zhang, X. Design and control of a powered hip exoskeleton for walking assistance. Int. J. Adv. Robot. Syst. 2015, 12, 18. [CrossRef]

14. Kong, K.; Jeon, D. Design and control of an exoskeleton for the elderly and patients. IEEE/ASME Trans. Mech. 2006, 11, 428-432. [CrossRef]

15. Hyon, S.; Morimoto, J.; Matsubara, T.; Noda, T.; Kawato, M. XoR: Hybrid drive exoskeleton robot that can balance. In Proceedings of the 2011 IEEE/RSJ International Conference on Intelligent Robots and Systems, San Francisco, CA, USA, 25-30 September 2011; pp. 3975-3981.

16. Nakamura, T.; Saito, K.; Wang, Z.; Kosuge, K. Realizing model-based wearable antigravity muscles support with dynamics terms. In Proceedings of the 2005 IEEE/RSJ International Conference on Intelligent Robots and Systems, Edmonton, AB, Canada, 2-6 August 2005; pp. 2694-2699.

17. Schiele, A. Ergonomics of exoskeletons: Subjective performance metrics. In Proceedings of the 2009 IEEE/RSJ International Conference on Intelligent Robots and Systems, St. Louis, MO, USA, 11-15 October 2009; pp. 480-485.

18. Cenciarini, M.; Dollar, A.M. Biomechanical considerations in the design of lower limb exoskeletons. In Proceedings of the 2011 IEEE International Conference on Rehabilitation Robotics, Zurich, Switzerland, 29 June-1 July 2011; pp. 1-6.

19. Park, Y.; Chen, B.; Young, D.; Stirling, L.; Wood, R.J.; Goldfield, E.; Nagpal, R. Bio-inspired active soft orthotic device for ankle foot pathologies. In Proceedings of the 2011 IEEE/RSJ International Conference on Intelligent Robots and Systems, San Francisco, CA, USA, 25-30 September 2011; pp. 4488-4495.

20. Park, Y.; Hen, B.C.; Perez-Arancibia, N.O.; Young, D.; Stirling, L.; Wood, R.J.; Goldfield, E.C.; Nagpal, R. Design and control of a bio-inspired soft wearable robotic device for ankle -foot rehabilitation. Bioinspir. Biomim. 2014, 9, 016007. [CrossRef] [PubMed]

21. Asbeck, A.T.; Rossi, S.M.M.D.; Holt, K.G.; Walsh, C.J. A biologically inspired soft exosuit for walking assistance. Int. J. Robot. Res. 2015, 34, 744-762. [CrossRef]

22. Asbeck, A.T.; Schmidt, K.; Galiana, I.; Wagner, D.; Walsh, C.J. Multi-joint soft exosuit for gait assistance. In Proceedings of the 2015 IEEE International Conference on Robotics and Automation, Seattle, WA, USA, 26-30 May 2015; pp. 6197-6204.

23. Ding, Y.; Galiana, I.; Asbeck, A.T.; Rossi, S.M.M.D.; Bae, J.; Santos, T.R.T.; Araujo, V.L.; Lee, S.; Holt, K.G.; Walsh, C. Biomechanical and Physiological Evaluation of Multi-joint Assistance with Soft Exosuits. IEEE Trans. Neural Syst. Rehabil. Eng. 2017, 25, 119-130. [CrossRef] [PubMed]

24. Jin, S.; Iwamoto, N.; Hashimoto, K.; Yamamoto, M. Experimental Evaluation of Energy Efficiency for a Soft Wearable Robotic Suit. IEEE Trans. Neural Syst. Rehabil. Eng. 2017, 25, 1192-1201. [CrossRef] [PubMed]

25. Jin, S.; Guo, S.; Hashimoto, K.; Xiong, X.; Yamamoto, M. Influence of a soft robotic suit on metabolic cost in long-distance level and inclined walking. Appl. Bionics Biomech. 2018, 2018, 9573951. [CrossRef]

26. Majidi, C. Soft robotics: A perspective current trends and prospects for the future. Soft Robot. 2014, 1, 5-11. [CrossRef]

27. Sekiya, N.; Nagasaki, H. Reproducibility of the walking patterns of normal young adults: Test-retest reliability of the walk ratio(step-length:step-rate). Gait Posture 1998, 7, 225-227. [CrossRef]

28. Shimada, H.; Hirata, T.; Kimura, Y.; Naka, T.; Kikuchi, K.; Oda, K.; Ishii, K.; Ishiwata, K.; Suzuki, T. Effects of a robotic walking exercise on walking performance in community-dwelling elderly adults. Geriatr. Gerontol. Int. 2009, 9, 372-381. [CrossRef]

29. Kikuuwe, R.; Yasukouchi, S.; Fujimoto, H.; Yamamoto, M. Proxy-based sliding mode control: A safer extension of PID position control. IEEE Trans. Robot. 2010, 26, 670-683. [CrossRef]

30. Holt, K.G.; Hamill, J.; Anders, R.O. Predicting the minimal energy costs of human walking. Med. Sci. Sport. Exerc. 1991, 23, 491-498. [CrossRef] 
31. Jin, S.; Guo, S.; Hashimoto, K.; Yamamoto, M. Influence of maximum assistive force of a soft wearable robotic suit on metabolic cost reduction. In Proceedings of the 8th IEEE International Conference on Cybernetics and Intelligent Systems and the 8th IEEE International Conference on Robotics, Automation and Mechatronics, Ningbo, China, 19-21 November 2017; pp. 146-150.

32. Rota, V.; Perucca, L.; Simone, A.; Tesio, L. Walk ratio (step length/cadence) as a summary index of neuromotor control of gait: Application to multiple sclerosis. Int. J. Rehabil. Res. 2011, 34, 265-269. [CrossRef] [PubMed]

33. Shimada, H.; Suzuki, T.; Kimura, Y.; Hirata, T.; Sugiura, M.; Endo, Y.; Yasuhara, K.; Shimada, K.; Kikuchi, K.; Oda, K.; et al. Effects of an automated stride assistance system on walking parameters and muscular glucose metabolism in elderly adults. Br. J. Sport. Med. 2011, 42, 922-929. [CrossRef]

34. Kerrigan, D.; Lee, L.; Collins, J. Reduced hip extension during walking: Healthy elderly and fallers versus young adults. Arch. Phys. Med. Rehabil. 2001, 82, 26-30. [CrossRef] [PubMed]

35. Cristopoliski, F.; Barela, J.; Leite, N.; Fowler, N.; Rodacki, A. Stretching exercise program improves gait in the elderly. Gerontology 2009, 55, 614-620. [CrossRef]

36. Kerrigan, D.; Xenopoulos-Oddsson, A.; Sullivan, M.; Lelas, J.; Riley, P. Effect of a hip flexor-stretching program on gait in the elderly. Arch. Phys. Med. Rehabil. 2003, 84, 1-6. [CrossRef]

37. Watt, J.; Jackson, K.; Franz, J.; Dicharry, J.; Evans, J.; Kerrigan, D. Effect of a supervised hip flexor stretching program on gait in frail elderly patients. PMER 2011, 3, 330-335.

38. Aboutorabi, A.; Arazpour, M.; Bahramizadeh, M.; Hutchins, S.; Fadayevatan, R. The effect of aging on gait parameters in able-bodied older subjects: A literature review. Aging Clin. Exp. Res. 2016, 28, 393-405. [CrossRef]

39. Kisner, C.; Colby, L. Therapeutic Exercise: Foundations and Techniques, 6th ed.; F. A Davis Company: Philadelphia, PA, USA, 2012.

40. Wert, D.; Brach, J.; Perera, S.; VanSwearingen, J. Gait biomechanics, spatial and temporal characteristics, and the energy cost of walking in older adults with impaired mobility. Phys. Ther. 2000, 90, 977-985. [CrossRef]

41. Waters, R.; Barnes, G.; Husserl, T.; Silver, L.; Liss, R. Comparable energy expenditure after arthrodesis of the hip and ankle. J. Bone Jt. Surg. Am. Vol. 1988, 70, 1032-1037. [CrossRef]

42. Donelan, J.; Kram, R. Mechanical and metabolic determinants of the preferred step width in human walking. Proc. R. Soc. Lond. Ser. B Biol. Sci. 2001, 268, 1985-1992. [CrossRef] [PubMed]

43. Gordon, K.E.; Ferris, D.P.; Kuo, A.D. Metabolic and mechanical energy costs of reducing vertical Center of Mass Movement During Gait. Arch. Phys. Med. Rehabil. 2009, 90, 136-144. [CrossRef] [PubMed]

44. Ellis, R.; Howard, K.; Kram, R. The metabolic and mechanical costs of step time asymmetry in walking. Proc. R. Soc. B Biol. Sci. 2013, 280, 1-7. [CrossRef] [PubMed]

(C) 2019 by the authors. Licensee MDPI, Basel, Switzerland. This article is an open access article distributed under the terms and conditions of the Creative Commons Attribution (CC BY) license (http://creativecommons.org/licenses/by/4.0/). 